\title{
DESENVOLVIMENTO DE UM DISPOSITIVO PARA A PREVENÇÃO DE ÚLCERA POR PRESSÃO EM CADEIRANTES
}

\author{
André Ferrus Filho \\ Graduado em Tecnólogo em processo de produção pelo Centro Universitário de Santo \\ André (1992). Especialista em Engenharia de Automação na Faculdade Mauá (2000) e \\ Administração da Produção FEI (2004), Mestre em Engenharia Biomédica na UMC \\ (2012). Professor da Faculdade de Tecnologia Termomecanica na área de mecatrônica. \\ andreffilho@terra.com.br \\ Marco Antonio Fumagalli
}

Graduado em Engenharia Mecânica pela EESC - USP (1988), tem um Nachdiplomstudium in Mechatronik (1990) e doutorado pelo Institute of Robotics da Eidgenössische Technische Hochschule - ETH Zurique (1998). Coordenador dos cursos de Engenharia da Universidade de Mogi das Cruzes (1998-2011).

fumagalli@umc.br

Núcio Elvino Mateus Theodório

Possui graduação em Educação Física pela Universidade de Mogi das Cruzes (1991). Graduação em Fisioterapia pela Universidade de Mogi das Cruzes (1995). Especialização em Ortopedia e Traumatologia pela Universidade do Grande ABC - UniABC (2001).

Mestrado em Engenharia Biomédica pela Universidade de Mogi das Cruzes (2005). nucio@hotmail.com

\section{Fabio Rubio}

Graduado em Tecnólogo em processo de fabricação pelo Centro Universitário de Santo André (2000), especialização em Estratégia para a Qualidade na FEI (2004) e Mestre em

Engenharia Biomédica na UMC (2012) Professor da Faculdade de Tecnologia

Termomecanica na área de mecatrônica industrial.

Fabio.rubio@terra.com.br

\section{RESUMO}

Úlceras por pressão ocorrem como consequência de uma obstrução de um vaso sanguíneo, devido à compressão do tecido sob a pele durante um longo período de tempo. Os indivíduos que usam cadeira de rodas ou estão imobilizadas na cama, e também as pessoas idosas com problemas de saúde têm uma grande tendência a desenvolver úlcera de pressão. O objetivo dessa pesquisa é desenvolver um assento eletromecânico que realize conjuntamente a inclinação látero-lateral e ântero-posterior adaptável em uma cadeira de rodas, e produza a alteração tanto da magnitude quanto da duração da pressão de interface. $\mathrm{O}$ assento em questão possui um sistema eletromecânico, que permite a movimentação do dispositivo em dois planos, sendo um frontal e outro lateral, acionados por motores DC, possibilitando assim avaliar a mudança de pressão de interface, e consequentemente podendo avaliar a evolução da patologia, em função da variação de pressão em tempos determinados. $\mathrm{O}$ equipamento desenvolvido suportou com facilidade uma pessoa adulta com aproximadamente $80 \mathrm{Kg}$ de massa na realização dos testes. Para possibilitar a coleta da pressão de interface, foi usado um equipamento sensorizado e controlado por um software para medir a pressão de interface entre superfícies de contato. A aquisição e o armazenamento dos ângulos de inclinação do assento móvel da cadeira foram realizados 
através do microcontrolador, programado em linguagem C. Para isso foi concebido um software para movimentar o dispositivo tanto através de um Joystick, como para posicionar o assento móvel em diferentes posições e intervalos de tempo diferentes. Resultados das medidas mostram uma redução da pressão média de interface em até $58 \%$. O equipamento, portanto, possibilitará futuras investigações no desenvolvimento das úlceras de pressão em cadeirantes, analisando tanto a alteração da magnitude quanto a duração da pressão de interface.

PALAVRAS-CHAVE: úlceras por pressão, interface de pressão, dispositivo de mudança de pressão, prevenção e controle.

\section{DEVELOPMENT OF A DEVICE TO PREVENT PRESSURE ULCERS IN WHEEL CHAIR}

\section{RESUMO}

Pressure ulcers occur as a result of an obstruction of a blood vessel due to compression of the tissue under the skin over a long period of time. Individuals who use a wheelchair or are immobilized in bed, and also older people with health problems have a tendency to develop pressure ulcers. The objective of this research is to develop an electromechanical seat to hold the slope together latero-lateral and anteroposterior adaptable in a wheelchair, and produces a change in both the magnitude and duration of interface pressure. The seat in question has an electromechanical system that allows moving the device in two planes, one front and one side, driven by DC motors, thus enabling to evaluate the change of interface pressure, and therefore can evaluate the evolution of the pathology, depending on pressure variation in time determined. The equipment developed bore easily with an adult about $80 \mathrm{~kg}$ mass on memory tests. To enable the collection of interface pressure, the equipment was used sensorized and controlled by software to measure the interface pressure between the contact surfaces. The acquisition and storage of the angles of inclination of the movable seat of the chair were performed using the microcontroller programmed in $\mathrm{C}$ language for a software that is designed to move the device through a joystick so as to move the movable seat in different positions and different time intervals. Results of the measurements show a reduction of pressure medium interface within 58\%. The equipment therefore enable future research on the development of pressure sores in wheelchair users, analyzing both the change in the magnitude and duration of interface pressure.

PALAVRAS-CHAVE: pressure ulcer, interface pressure, device to weight distribution and prevention and control.

\section{DESENVOLVIMENTO DE UM DISPOSITIVO PARA A PREVENÇÃO DE ÚLCERA POR PRESSÃO EM CADEIRANTES}

\section{INTRODUÇÃO}

As úlceras de pressão (UP) são áreas localizadas de isquemia e necrose tecidular, que se desenvolvem pela compressão prolongada dos tecidos moles entre proeminências ósseas e 
a superfície externa (ROCHA, et al 2006). Essas lesões são provocadas pela alteração do reflexo de dor em pacientes com lesão medular (tetraplégicos, paraplégicos ou hemiplégicos) ou pacientes debilitados, idosos ou cronicamente doentes (COSTA et al 2006). Vários são os termos utilizados inadequadamente para as úlceras de pressão, tais como úlcera de decúbito, úlcera de acamado, úlceras isquêmicas e escaras. Esses termos não descrevem adequadamente a fisiopatologia da úlcera de pressão (COSTA et al 2006) . As úlceras de pressão adquiridas em locais de tecido colapsado por pressão ocorrem principalmente sob as tuberosidades isquiáticas (TI) (GEFFEN et al, 2009). Essas lesões ocorrem em indivíduos que usam cadeira de rodas ou que estão restritos ao leito. Ao permanecer em uma só postura por muito tempo o apoio de sustentação causa uma pressão local.

Reenalda (2009) fez uma grande revisão sistemática dos artigos, analisando a relação entre pressão de interface e o desenvolvimento de úlceras de pressão ou sua cura. A maioria destes artigos demonstra uma relação direta entre a pressão de interface e o desenvolvimento de úlceras de pressão, entretanto não se pôde relacionar as características pessoais, os aspectos temporais da pressão de interface e o surgimento de úlceras de pressão, assim como não há como quantificar os limiares de interface de pressão, ficando uma análise qualitativa e não quantitativa.

Geffen (2009) projetou uma cadeira simuladora que permite estudar os movimentos independentes, do tronco da pelve e das coxas. Os estudos tiveram a finalidade de alinhar os eixos angulares da cadeira com os eixos de rotação dos respectivos segmentos do corpo alternando diferentes posições e verificar esse comportamento em indivíduos que não podem funcionalmente reposicionar-se e adotam uma postura passiva. Os resultados mostram que é necessário fazer uma melhor avaliação a fim de visar à carga corporal em função da postura sentada na cadeira de rodas. Para calcular esta carga do corpo sobre a região de apoio na cadeira é essencial sabermos como ajustes angulares influenciam na orientação do corpo em relação à cadeira.

Springle (2011) fez uma revisão de vários artigos sobre a redistribuição da pressão para a prevenção de úlceras de pressão. Em seu artigo ele mostra que a magnitude da pressão depende dos tipos das superfícies de apoio, bem como a postura corporal no apoio das superfícies. A duração da pressão de interface depende da mudança e do deslocamento do peso, bem como do uso de superfícies dinâmicas que redistribuem ativamente as pressões de interface. Este artigo mostra que as intervenções preventivas devem ser direcionadas para ambos os casos como: magnitude e duração da pressão. Neste artigo, é mostrado também que usuários de cadeiras de rodas tem a alta propensão a desenvolver úlcera de pressão, principalmente os que não podem se mover de forma independente para produzir uma variação de pressão na interface. Existem algumas recomendações para usuários de cadeiras de rodas com lesão da medula espinal (SCI) alterem devem alterar a sua posição a cada hora durante 60 segundos, porém muitos estudos ainda devem ser realizados.

Em face do exposto, existe ainda uma grande investigação a ser realizada face o desenvolvimento das úlceras de pressão em cadeirantes, que deve focar tanto na alteração da magnitude quanto na duração da pressão de interface.

Em virtude destes estudos o objetivo deste trabalho foi desenvolver um assento eletromecânico robusto e que realize conjuntamente a inclinação látero-lateral e ânteroposterior, tanto manual quanto programável, que seja facilmente adaptável em uma cadeira de rodas, tenha facilidade de transporte e produza a alteração tanto da magnitude quanto da duração da pressão de interface. E desta forma possa mensurar as pressões exercidas sobre o assento nas regiões das tuberosidades isquiáticas por meio de sensores de pressão, em pessoas saudáveis durante as inclinações, a fim de investigar a variação de pressão em toda região. 


\section{DISPOSITIVOS PARA PREVENÇÃO DAS ÚLCERAS POR PRESSÃO}

Existem varias técnicas para a melhora da distribuição de pressão sobre a base do assento. Brienza et al, (1996) mostra uma técnica que consiste em monitorar em tempo real a modificação do formato da superfície de suporte e seu efeito sobre a pressão de contato, com o uso de um arranjo de atuadores e sensores integrados a um computador que fornece a forma interativa até que se obtenham gradientes de pressão suavemente distribuídos. Este sistema é denominado CASS, conforme ilustrado na figura 1. Nesta técnica, representações matemáticas da anatomia das nádegas e coxas humanas foram propostas e testadas, e mostraram-se válidas na análise e no poder preditivo do efeito das pressões mecânicas sobre essa região anatômica.

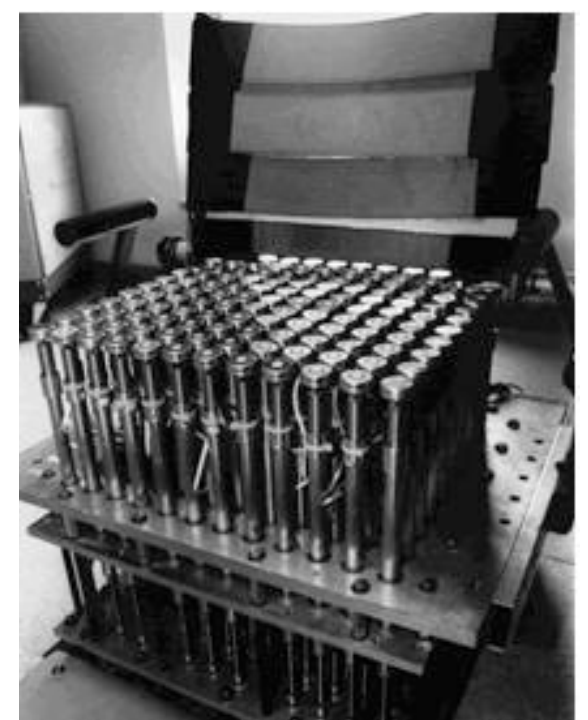

\section{Figura1- Sistema de Assento Automático Computadorizado CASS}

Almofadas como ilustradas na figura 2 com diferentes tipos de fluidos são utilizados como prevenção e controle das úlceras de pressão, para indivíduos acamados e ou usuários de cadeira de rodas, para permitir a distribuição da massa corpórea e minimizar a pressão máxima de interface sobre as proeminências ósseas, como é o caso dos colchões com água, gel e ar.

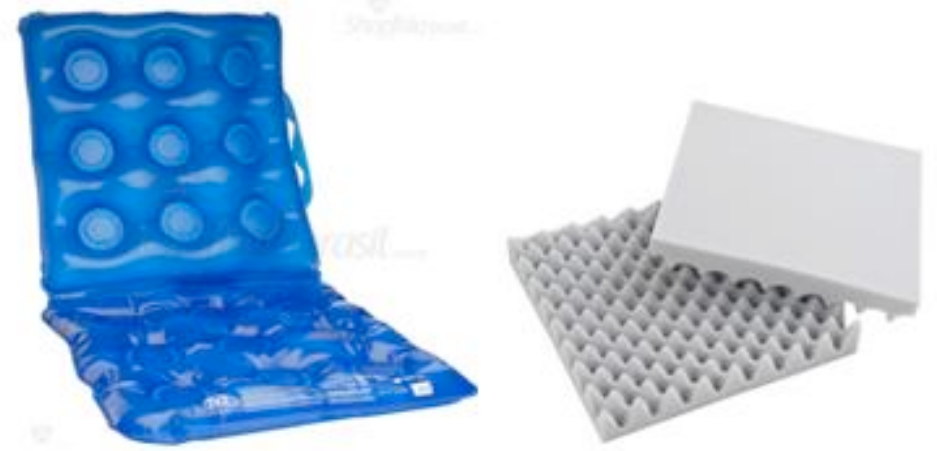

\section{Figura 2 - Tipos de Almofadas usadas na Prevenção de Úlceras por Pressão}

Muitos estudos buscam correlacionar os valores das pressões na interface com o possível aparecimento das úlceras de pressão em pessoas que permanecem por muito tempo sentado na mesma posição. 
Os estudos analisados apresentam grande heterogeneidade na amostragem, como falta de padronização do protocolo de pesquisa, da definição e medição do prognóstico e dos fatores preditivos, o que limita o comparativo dos diferentes estudos, gerando uma análise e validação enfraquecida. Embora sejam observadas melhoras nas técnicas de medição, ainda há deformação nos resultados, dificultando a análise dos dados obtidos. De acordo com os resultados obtidos sobre a relação entre pressão de interface e desenvolvimento e cura de úlceras de pressão, as pressões mais elevadas de interface parecem estar relacionadas com uma maior incidência de úlceras de pressão. Dois estudos demonstraram que um aumento da pressão de interface é relacionado a uma maior incidência de úlceras de pressão. A relação entre a pressão de interface com o desenvolvimento de úlcera de pressão foi definida de forma retrospectiva. No entanto, faltava qualidade metodológica a este estudo para obter uma conclusão. Em outros três estudos não houve relação entre pressão de interface e o desenvolvimento ou a cura de úlceras de pressão.

Os estudos relacionados a interface de pressão incluídos na presente revisão, têm usado uma variedade de dispositivos diferentes para medir, mas de modo geral, não ficam claras as descrições das medições. Além disso, a precisão e a estabilidade dos dispositivos de medição da pressão são limitadas.

Tem-se observado que os dispositivos de medição de pressão apresentam histerese substancial e deformação, embora a técnica de medição de pressão esteja melhorando. Como resultado, a quantificação e a normalização da carga são difíceis.

Este estudo poderia explicar algumas das diferenças encontradas entre os estudos e porque os valores de pressão subseqüentes não podem ser comparados facilmente. Entidades envolvidas no assunto estão atualmente trabalhando na padronização das técnicas de medição para a pressão de interface. Essa padronização vai facilitar a comparação dos dados entre diferentes estudos.

\section{LIMITAÇÕES DAS TÉCNICAS DE PREVENÇÃO}

Segundo Kanj et al 1998, a cicatrização das úlceras de pressão é baixa, resultando em alta mortalidade. Alguns estudos mostram que 50 a $70 \%$ das úlceras de pressão não são cicatrizadas após a intervenção cirúrgica em pacientes paraplégicos. Mesmo com a aplicação de procedimentos de prevenção, a cicatrização das úlceras de pressão é considerada baixa. Indivíduos que apresentam déficit sensorial, mobilidade limitada e falência de órgãos causados por problemas neurológicos e ou pulmonar dificultam o controle da evolução das úlceras de pressão.

Os processos de técnicas de prevenção como: monitoramento com inspeção visual e utilização de almofadas nos casos de pacientes usuários de cadeira de rodas com saúde debilitada, com déficit sensorial e ou até com necrose do tecido da pele são casos em que a busca por resultados que possibilitem a ausência e ou diminuição da evolução da patologia não ocorra com sucesso na maioria dos casos. Por outro lado, resulta em aumento dos custos de manutenção das técnicas utilizadas.

Atualmente o custo de manutenção com inspeção visual e utilização de almofadas é considerado menor, se comparado com as sucessivas intervenções cirúrgicas a que um paciente com úlcera por pressão é submetido, porém, ainda pode-se dizer que o custo de manutenção com inspeção visual é relativamente maior do que os custos com a utilização de sistemas de apoio da massa corpórea, tais como almofadas com fluído estático e dinâmico.

A classificação ou estágios de evolução das úlceras de pressão, os tipos de dispositivos utilizados para a prevenção, as avaliações das pressões críticas e atuantes em diversas 
posições do corpo humano sobre as superfícies de sustentação, fatores físiológicos e físicos, técnicas de prevenção e suas limitações, bem como as técnicas de tratamento foram consideradas como referência no desenvolvimento de nosso trabalho de pesquisa.

Sendo assim, podemos considerar este estudo como o precursor em utilizar um dispositivo eletromecânico para promover a inclinação do assento de apoio e sustentação de uma cadeira, resultando na mudança das pressões de interface.

\section{METODOLOGIA}

Muitas são as dificuldades em prevenir a presença e ou a evolução de uma úlcera de pressão em usuários que permanecem por muito tempo sentado em cadeira de rodas. Portanto fazer o deslocamento deste peso sobre o apoio e sustentação da massa corpórea sobre uma cadeira possibilitará a distribuição do peso sobre as superfícies de contato, bem como irá resultará na mudança e diminuição das pressões de interface nas regiões onde ocorre alívio da massa corporal.

Considerando que o usuário quando sentado sobre uma cadeira de rodas inicialmente está com o tronco na posição ereta, propor o deslocamento ou inclinação do tronco em relação ao eixo vertical central a que o usuário se encontra resulta no deslocamento do centro de gravidade e do peso sobre o assento da cadeira.

Baseado nesta hipótese propõe-se desenvolver um sistema eletromecânico que proporcione a inclinação do peso do usuário sobre o assento de uma cadeira, com e sem almofada, mediante o deslocamento vertical dos planos lateral e frontal do assento de apoio e sustentação de uma cadeira.

A movimentação da plataforma é executada através de um joystick, no qual os ângulos de inclinação deste assento são projetados para deslocar em quatro direções: lateral direita, lateral esquerda e frente ou para trás. $\mathrm{O}$ microcontrolador PIC é responsável por receber a informação do joystick e decidir, de acordo com o software desenvolvido em linguagem $C$, qual é a direção e o sentido de movimentação requisitado pelo usuário. Também tem a função de aquisição do sinal dos potenciômetros de realimentação e através de equações específicas, o relaciona com o ângulo de seu respectivo plano mostrando o resultado em um display alfanumérico.

\section{DESENVOLVIMENTO DO DISPOSITIVO}

Para atingir o objetivo proposto desta pesquisa e possibilitar a inclinação do assento de apoio e sustentação de um indivíduo com até $100 \mathrm{Kg}$ de massa na posição sentada, foram desenvolvidas duas plataformas com as dimensões de $350 \times 350 \times 25 \mathrm{~mm}$ que podem ser adaptadas em uma cadeira de rodas articulável como mostrado na figura 3. A movimentação deste dispositivo nos dois planos é realizada por dois motores de $100 \mathrm{~W}$ cada, com respectivos redutores. Um pivô central tipo uniball, é posicionado no centro do dispositivo, possibilitando assim a movimentação do dispositivo através de um sistema de articulação em dois planos. A plataforma tem um limite mecânico em seu deslocamento angular de $16^{\circ}$, sendo no máximo de $8^{\circ}$ para cada lado, e realiza a movimentação em duas direções independentemente. Esta plataforma tem características dimensionais semelhantes ao assento de uma cadeira, podendo assim ser facilmente adaptado a qualquer cadeira de rodas articulável. 


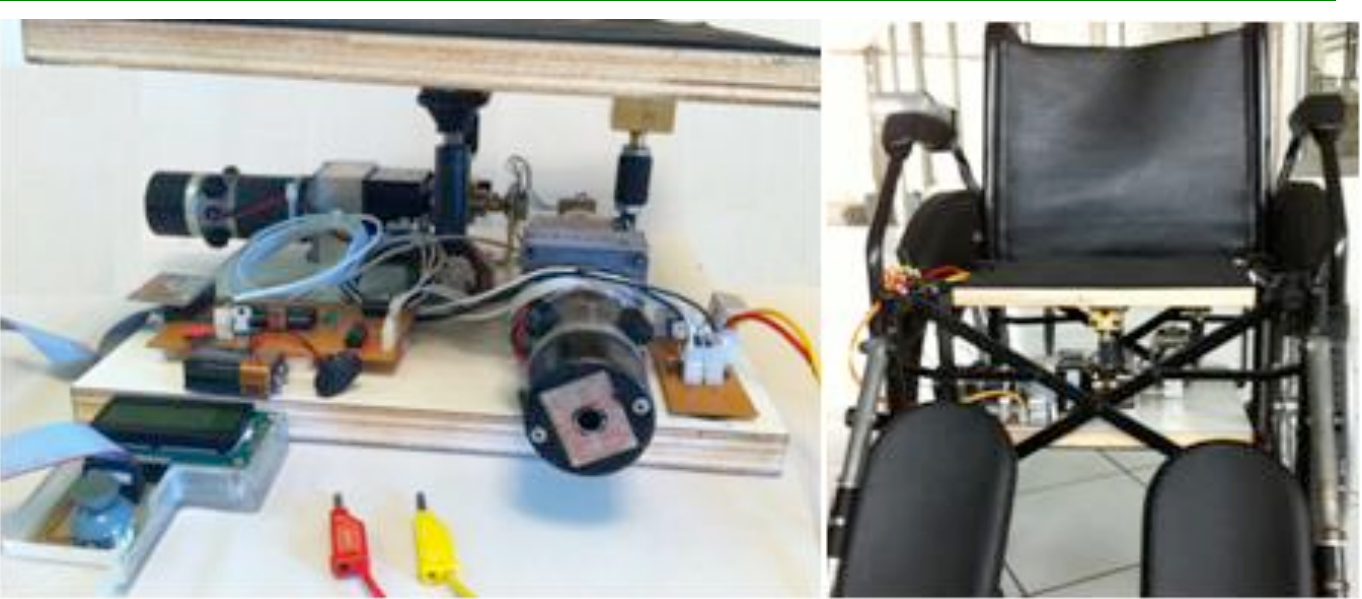

Figura 3 - Dispositivo Montado na Cadeira

\section{PROCEDIMENTOS PARA COLETA DE DADOS}

A coleta das medidas da pressão de interface sobre as superfícies de contato, entre a plataforma do assento móvel e o tecido da pele da região glútea, precisamente na região das tuberosidades isquiáticas, foi realizada utilizando um baropodômetro da marca FootWork, com peso de $3 \mathrm{~kg}$, e dimensões de 654 x 520 × $25 \mathrm{~mm}$. Sua superfície ativa é de $400 \times 400 \times 05 \mathrm{~mm}$ de espessura. Trabalha com freqüência de $150 \mathrm{~Hz}$ e conversor analógico de 16 bits, sua pressão máxima por captador é de $100 \mathrm{~N} / \mathrm{cm} 2$. Possui 2.704 captadores capacitivos calibrados com dimensões de 7.62 × $7.62 \mathrm{~mm}$. Após o individuo posicionar-se sentando sobre a placa, os valores são enviados automaticamente para um software próprio de aquisição dados, onde a superfície de atuação é graficamente demonstrada na tela do computador.

O plano frontal (PF) é definido como a posição de inclinação do assento móvel para frente e para trás, tomando como ponto de referência a vista frontal da cadeira. O plano lateral (PL) ocorre perpendicular ao plano frontal onde a inclinação fica a direita e para a esquerda conforme pode ser apresentado na figura 4. Os movimentos ocorrem, respectivamente, em relação ao eixo central da estrutura do assento móvel, que corresponde a $153 \mathrm{~mm}$. Esta distância é considerada como valor fixo para a identificação dos ângulos de inclinação $\beta$ no plano lateral e $\alpha$ no plano frontal do assento móvel, utilizando-se uma equação trigonométrica, conforme apresentado na figura 4.

Para a identificação e registro do ângulo de inclinação do assento móvel, foi utilizado um sistema de leitura através do display LCD que indica a posição do ângulo e de um joystick para mover o dispositivo na posição desejada.
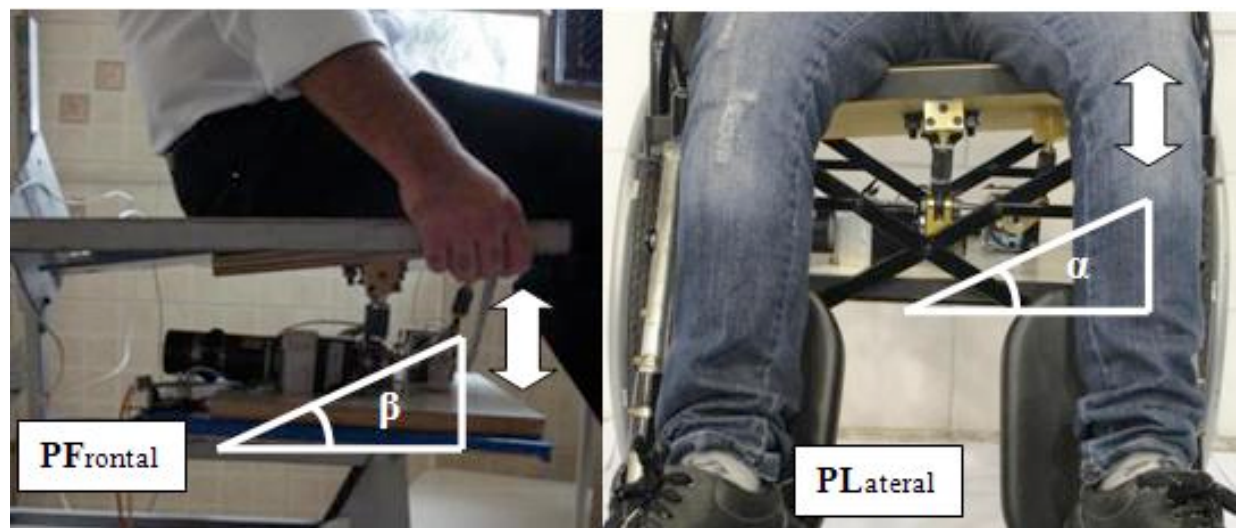

Figura 4 - Plano Frontal e Plano Lateral da Cadeira 


\section{RESULTADOS E DISCUSSÃO}

Os valores medidos da pressão de interface, durante a inclinação do assento móvel da cadeira, são apresentados a seguir. Para análise das medidas das pressões de interface obtidas durante os testes, será verificado o aumento ou redução da pressão na região capturada pelo software.

O equipamento foi calibrado na posição neutra com ângulo de $0^{\circ}$. Os valores de pressões estão relacionados com a variação de cores do gráfico, no qual o azul denota valores menores e o vermelho corresponde aos maiores valores.

Os resultados mostram a variação de pressão e a variação do centro de massa, à medida que a plataforma vai se inclinando em cada direção.

Durante as medições observou-se que com ângulos inferiores a $3^{\circ}$ não houve alterações significativas nas pressões de interface, por este motivo as medidas foram registradas somente a partir do ângulo de $3^{\circ}$.

Sendo assim a primeira medição foi realizada no plano lateral (PL) usando como referência um ângulo mínimo de $3^{\circ}$ e um ângulo máximo de $8^{\circ}$ à direita como mostra a figura $5 \mathrm{com}$ os seus respectivos valores ilustrados na tabela 1 . O mesmo ensaio foi realizado posteriormente à esquerda como mostra a figura 6 , com os valores do ensaio ilustrados na tabela 2. Estes mesmos testes foram repetidos no plano frontal (PF) com ângulos de $3^{\circ}$ e $8^{\circ}$ para trás, representado na figura 7 em conjunto com a tabela 3 . Porém no plano frontal para frente foi realizado um teste somente com ângulo de $3^{\circ}$ ilustrado na figura $8 \mathrm{com}$ dados na tabela 4 , pois com ângulo de $8^{\circ}$, o voluntário não conseguiu se equilibrar, impossibilitando o software de fazer a leitura. Observa-se que o limite mecânico da plataforma de seu deslocamento angular é de $16^{\circ}$, sendo, portanto, no máximo de $8^{\circ}$ para cada lado.

O software utilizado nos fornece, na tela através dos dados coletados, a pressão máxima (PMax), pressão média (PM) e a pressão mínima (PMin) do lado esquerdo (E) e direito (D) dos glúteos. Os valores obtidos pelo software são dados em $\mathrm{Kgf} / \mathrm{cm} 2$, sendo que 1 $\mathrm{Kgf} / \mathrm{cm} 2=735.56 \mathrm{~mm} / \mathrm{Hg}$.

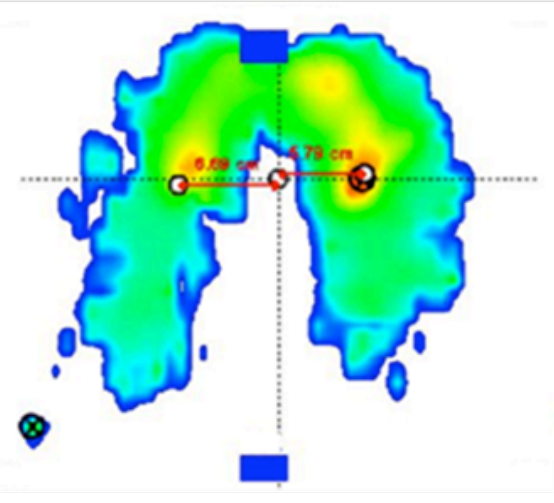

Posição

Neutra Ângulo

$0^{\circ}$

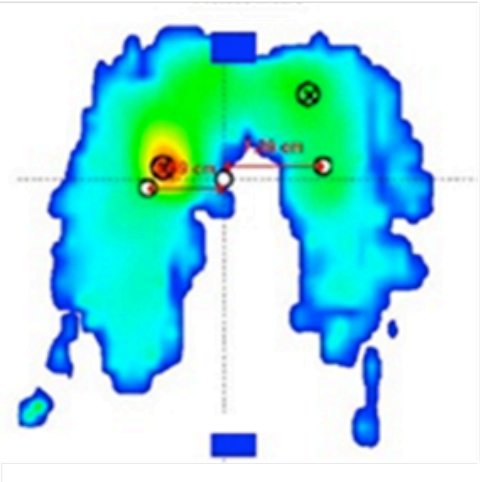

Posição PL Ângulo $3^{\circ}$

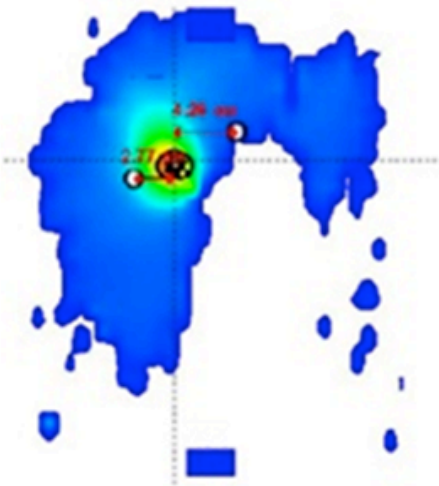

Posição PL Ângulo $8^{\circ}$

Figura 5 - Pressão de Interface no Plano Lateral Direito 
Tabela 1: Valores das Medições no Plano Lateral Direito

\begin{tabular}{|l|c|c|c|c|c|c|c|c|}
\hline & \multicolumn{7}{|c|}{ Movimentação no Plano Lateral à Direita } \\
\hline \multirow{2}{*}{$\begin{array}{c}\text { Pressão } \\
\left(\mathrm{Kgf} / \mathrm{cm}^{2}\right)\end{array}$} & \multicolumn{5}{|c|}{ Direito } & Var \% & \multicolumn{3}{c|}{ Esquerdo } & Var \% \\
\cline { 2 - 9 } & $0^{\circ}$ & $\mathbf{3}^{\circ}$ & $8^{\circ}$ & & $0^{\circ}$ & $3^{\circ}$ & $\mathbf{8}^{\circ}$ & \\
\hline $\mathrm{P}_{\text {maxima }}$ & 0,30 & 0,43 & 1,60 & 600 & 0,30 & 0,19 & 0,10 & $\mathbf{2 0 0}$ \\
\hline $\mathrm{P}_{\text {minima }}$ & 0,08 & 0,11 & 0,17 & $\mathbf{1 1 2}$ & & & & \\
\hline $\mathrm{P}_{\text {media }}$ & & & & & 0,12 & 0,09 & 0,07 & $\mathbf{5 8}$ \\
\hline
\end{tabular}

A PMáx. do lado direito variando de $0^{\circ}$ a $8^{\circ}$ obteve um aumento de $600 \%$, a PMin apresentou um aumento de $112 \%$. No entanto do lado esquerdo a PMédia sofreu uma redução de $58 \%$ e a PMax. atingiu uma redução de $200 \%$.

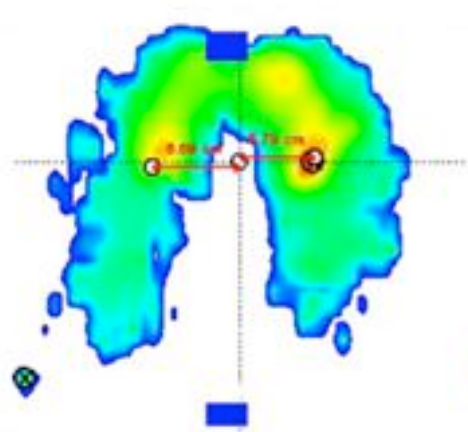

Posição Neutra

Ângulo $0^{\circ}$

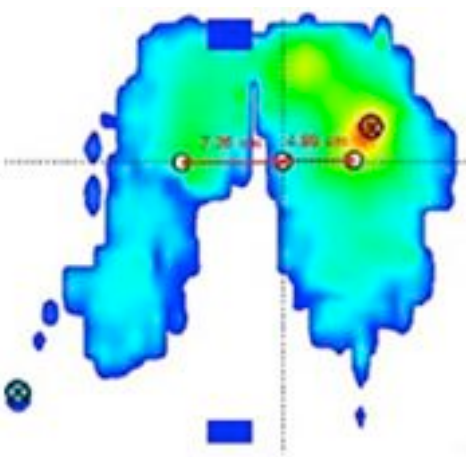

Posição PL

Ângulo $3^{\circ}$

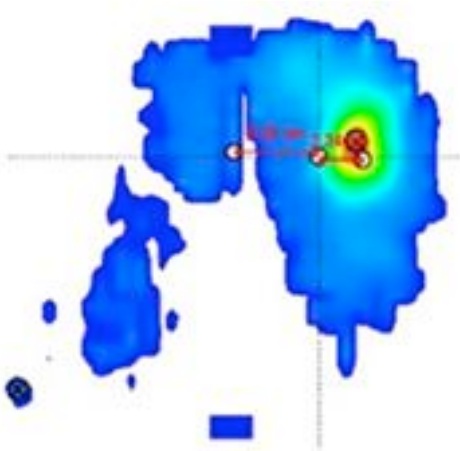

Posição PL

Ângulo $8^{\circ}$

Figura 6 - Pressão de Interface no Plano Lateral Esquerdo

Tabela 2: Valores das Medições no Plano Lateral Esquerdo

\begin{tabular}{|l|c|c|c|c|c|c|c|c|}
\hline & \multicolumn{7}{|c|}{ Movimentação no Plano Lateral à Esquerda } \\
\hline $\begin{array}{c}\text { Pressão } \\
\left(\mathbf{K g f} / \mathbf{c m}^{2}\right)\end{array}$ & \multicolumn{3}{|c|}{ Direito } & Var \% & \multicolumn{3}{c|}{ Esquerdo } & Var \% \\
\cline { 2 - 9 } & $\mathbf{0}^{\circ}$ & $\mathbf{3}^{\circ}$ & $\mathbf{8}^{\circ}$ & & $\mathbf{0}^{\circ}$ & $\mathbf{3}^{\circ}$ & $\mathbf{8}^{\circ}$ & \\
\hline $\mathbf{P}_{\text {maxima }}$ & 0,30 & 0,19 & 0,11 & $\mathbf{- 2 0 0}$ & 0,30 & 0,44 & 1,59 & $\mathbf{6 0 0}$ \\
\hline $\mathbf{P}_{\text {minima }}$ & & & & & 0,09 & 0,12 & 0,19 & $\mathbf{1 1 3}$ \\
\hline $\mathbf{P}_{\text {media }}$ & 0,11 & 0,09 & 0,07 & $\mathbf{- 5 7}$ & & & & \\
\hline
\end{tabular}

A PMáx. do lado esquerdo variando de $0^{\circ}$ a $8^{\circ}$ obteve um aumento de $600 \%$, a PMin apresentou um aumento de $113 \%$. No entanto do lado direito a PMédia sofreu uma redução de $57 \%$ e a PMax atingiu uma redução de $200 \%$. 


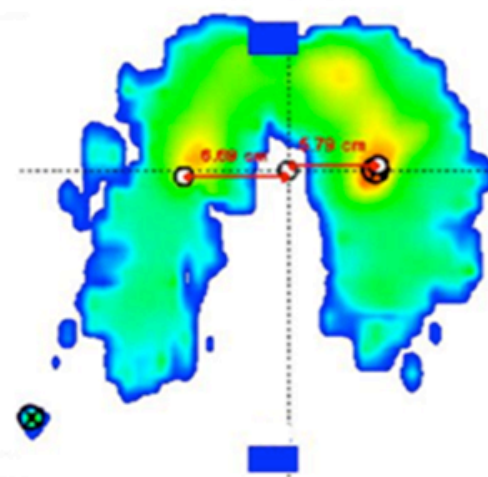

Posição

Neutra Ângulo $0^{\circ}$

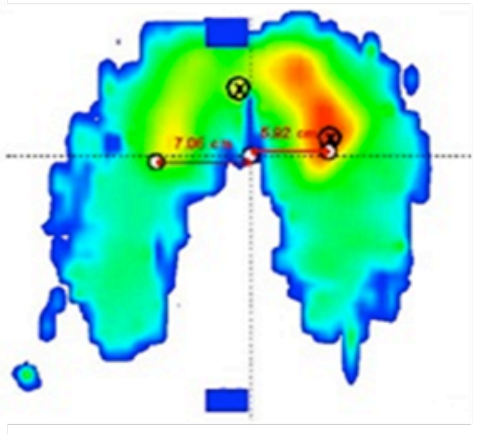

Posição PF

Ângulo $3^{\circ}$

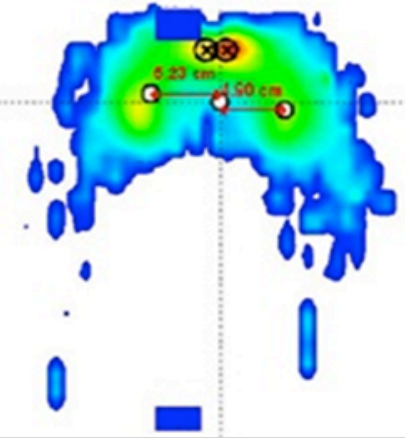

Posição PF Ângulo $8^{\circ}$

Figura 7 - Pressão de Interface no Plano Frontal para Trás

Tabela 3: Valores das Medições no Plano Frontal para Trás

\begin{tabular}{|l|c|c|c|c|c|c|c|c|}
\hline & \multicolumn{7}{|c|}{ Movimentação no Plano Frontal para trás } \\
\hline \multirow{2}{*}{$\begin{array}{c}\text { Pressão } \\
\left(\mathrm{Kgf} / \mathrm{cm}^{2}\right)\end{array}$} & \multicolumn{3}{|c|}{ Parte de Trás } & Var \% & \multicolumn{3}{c|}{ Parte da Frente } & Var $\%^{\circ}$ \\
\cline { 2 - 9 } & $\mathbf{0}^{\circ}$ & $\mathbf{3}^{\circ}$ & $\mathbf{8}^{\circ}$ & & $0^{\circ}$ & $\mathbf{3}^{\circ}$ & $\mathbf{8}^{\circ}$ & \\
\hline $\mathrm{P}_{\text {maxima }}$ & 0,30 & 0,33 & 0,52 & $\mathbf{7 4}$ & 0,30 & 0,19 & 0,12 & $\mathbf{1 5 0}$ \\
\hline $\mathrm{P}_{\text {minima }}$ & 0,08 & 0,09 & 0,10 & $\mathbf{2 5}$ & & & & \\
\hline $\mathrm{P}_{\text {média }}$ & & & & & 0,19 & 0,19 & 0,08 & $\mathbf{4 2}$ \\
\hline
\end{tabular}

A PMáx. para trás de $0^{\circ}$ a $8^{\circ}$ obteve um aumento de $74 \%$, a PMin apresentou um aumento de $25 \%$. No entanto na parte da frente a PMédia sofreu uma redução de $42 \%$ e a PMax atingiu uma redução de $150 \%$.

Figura 8 - Pressão de Interface no Plano Frontal para Frente

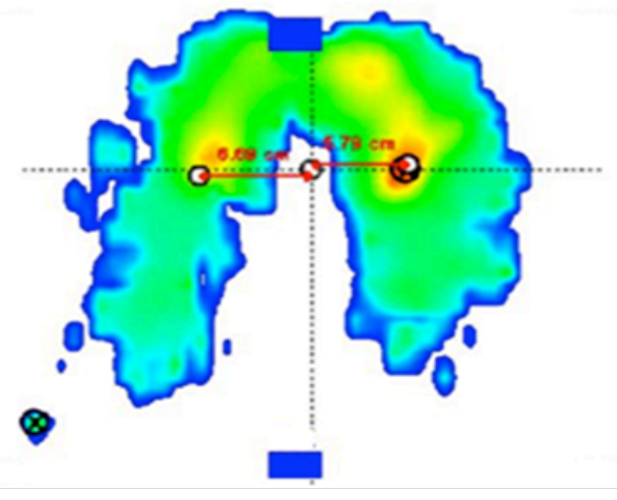

Posição

Neutra Ângulo $0^{\circ}$

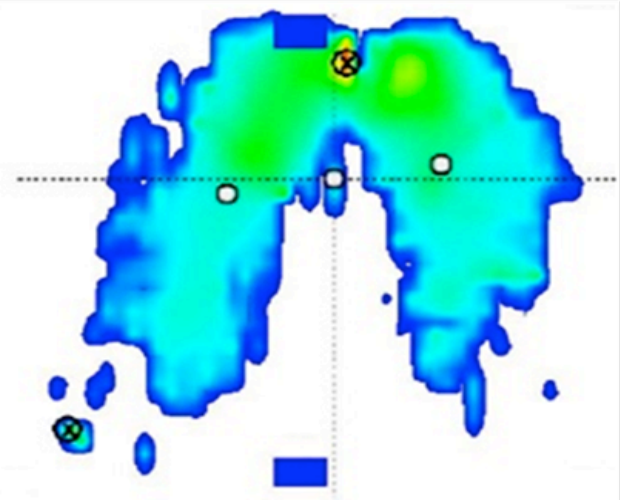

Posição PF

Ângulo $3^{\circ}$ 
Tabela 4: Valores das Medições no Plano Frontal para Frente

\begin{tabular}{|l|c|c|c|c|c|c|c|c|}
\hline & \multicolumn{7}{|c|}{ Movimentação no Plano Frontal para Frente } \\
\hline \multirow{2}{*}{$\begin{array}{c}\text { Pressão } \\
\left(\mathrm{Kgf}_{\mathrm{g}} \mathrm{cm}^{2}\right)\end{array}$} & \multicolumn{3}{|c|}{ Parte de Trás } & Var \% & \multicolumn{3}{c|}{ Parte da Frente } & Var\% \\
\cline { 2 - 9 } & $0^{\circ}$ & $\mathbf{3}^{\circ}$ & $\mathbf{8}^{\circ}$ & & $0^{\circ}$ & $\mathbf{3}^{\circ}$ & $\mathbf{8}^{\circ}$ & \\
\hline $\mathrm{P}_{\text {maxima }}$ & 0,30 & 0,45 & & $\mathbf{5 0}$ & 0,19 & 0,14 & & $\mathbf{3 6}$ \\
\hline $\mathrm{P}_{\text {minima }}$ & 0,08 & 0,08 & & $\mathbf{0}$ & & & & \\
\hline $\mathrm{P}_{\text {média }}$ & & & & & 0,12 & 0,09 & & $\mathbf{2 5}$ \\
\hline
\end{tabular}

A PMáx. para trás de $0^{\circ}$ a $3^{\circ}$ obteve um aumento de $50 \%$, a PMin não sofreu alteração. No entanto na parte da frente a PMédia sofreu uma redução de $25 \%$ e a PMax atingiu uma redução de $36 \%$.

\section{CONSIDERAÇÕES FINAIS}

A plataforma possui características e dimensões que se encaixam facilmente em uma cadeira de rodas articulável ou possa ser utilizado em qualquer superfície rígida. $\mathrm{O}$ mecanismo possibilita a realização dos movimentos de inclinação do assento de apoio e sustentação da massa corpórea de um indivíduo, na posição sentada.

O sistema desenvolvido mostrou-se bem robusto, foi capaz de suportar a massa de pessoas com até $100 \mathrm{~kg}$ e de permitir a inclinação do assento em torno do eixo central sem dificuldades mecânicas ou elétricas. Com carga, o equipamento consume uma baixa corrente em torno de 0,8A (ampére). Outro ponto relevante foi alimentação, pois o mesmo necessita apenas de uma bateria de $12 \mathrm{~V}$ (volts) para funcionar, tornando o dispositivo fácil de ser transportado.

Para possibilitar a coleta de dados neste estudo, foi usado um equipamento sensorizado e controlado por um software para medir a pressão de interface entre superfícies de contato. O sensor de pressão mostrou alta sensibilidade e homogeneidade na realização das medidas. Foi usado também um potenciômetro e um microcontrolador programado em linguagem $\mathrm{C}$, para medir deslocamento angular.

De acordo com os testes realizados neste estudo, podemos afirmar que o dispositivo desenvolvido atingiu seu objetivo, pois durante a realização dos testes todo conjunto mostrou-se bastante confiável.

Os resultados obtidos mostraram uma redução significativa da pressão média e da pressão máxima na região de interface quando o indivíduo foi submetido à inclinação do assento em diversas posições. $\mathrm{O}$ melhor resultado ocorreu com a mudança lateral do equipamento em um ângulo de $8^{\circ}$, havendo uma redução de até $58 \%$ na pressão média e de $200 \%$ na pressão máxima do lado em que houve o alívio.

\section{REFERÊNCIAS BIBLIOGRÁFICAS}

1. BRIENZA D.M. et al. A system for the analysis of seat support surfaces using surface shape control and simultaneous measurement of applied pressure. IEEE Transactions on Neural System and Rehabilitation Engineering, 1996; v.4, n.2, p.103-113, 1996. 
2. COSTA, M. P. et al. Epidemiologia e tratamento das úlceras de pressão: Experiência de 77 Casos. Acta Ortopédica Brasileira, v. 13, n. 3, p. 128-129, 2006.

3. GEFFEN, P. VAN, Dynamic Sitting. Tese (doutorado em Engenharia Biomédica) Universidade Twente, Amsterdam Holanda, 2009.

4. GEFFEN, P. VAN et al. The effects of a dynamic tuberal support on ischial buttock load and pattern of blood supply. Transactions on Neural Systems and Rehabilitation Engineering, v. 18, n. 1, fev. 2009.

5. KANJ, L. F. et al. Continuing medical education. Journal of the American Academy of Dermatology, v. 38, p. 517-532, jun. 1998.

6. REENALDA, J. Dynamic Sitting to Prevent Pressure Ulcers in Spinal Cord Injured. Tese (doutorado em Engenharia Biomecânica) Universidade Twente, Holanda, 2009.

7. ROCHA, J. A. et al. Abordagem Terapêutica das Úlceras de Pressão: Intervenções Baseadas na Evidência. Acta Médica Portuguesa; v. 19: p. 29-38, 2006.

8. SPRIGLE, S. et al. Assessing evidence supporting redistribution of pressure for pressure ulcer prevention, Journal of Rehabilitation Research \& Development, v. 48 n. 3, p. 203-14, 2011. 